\title{
Clinical investigation of TROP-2 as an independent biomarker and potential therapeutic target in colon cancer
}

\author{
PENG ZHAO $^{1}$, HAI-ZHENG YU ${ }^{2}$ and JIAN-HUI CAI ${ }^{1}$ \\ ${ }^{1}$ Department of Surgery, Hebei Medical University, Shijiazhuang, Hebei 050017; ${ }^{2}$ Department of Social Sciences, \\ Hebei United University, Tangshan, Hebei 063000, P.R. China \\ Received July 12, 2014; Accepted May 6, 2015
}

DOI: $10.3892 / \mathrm{mmr} .2015 .3900$

\begin{abstract}
Colon cancer is associated with a severe demographic and economic burden worldwide. The pathogenesis of colon cancer is highly complex and involves sequential genetic and epigenetic mechanisms. Despite extensive investigation, the pathogenesis of colon cancer remains to be elucidated. As the third most common type of cancer worldwide, the treatment options for colon cancer are currently limited. Human trophoblast cell-surface marker (TROP-2), is a cell-surface transmembrane glycoprotein overexpressed by several types of epithelial carcinoma. In addition, TROP-2 has been demonstrated to be associated with tumorigenesis and invasiveness in solid types of tumor. The aim of the present study was to investigate the protein expression of TROP-2 in colon cancer tissues, and further explore the association between the expression of TROP-2 and clinicopathological features of patients with colon cancer. The expression and localization of the TROP-2 protein was examined using western blot analysis and immunofluorescence staining. Finally, the expression of TROP-2 expression was correlated to conventional clinicopathological features of colon cancer using a $\chi^{2}$ test. The results revealed that TROP-2 protein was expressed at high levels in the colon cancer tissues, which was associated with the development and pathological process of colon cancer. Therefore, TROP-2 may be used as a biomarker to determine the clinical prognosis, and as a potential therapeutic target in colon cancer.
\end{abstract}

Correspondence to: Dr Jian-Hui Cai, Department of Surgery, Hebei Medical University, 361 East Zhongshan Road, Shijiazhuang, Hebei 050017, P.R. China

E-mail: caijianhui1970@yeah.net

Abbreviations: CC, colon cancer; CRC, colorectal cancer; TROP-2, trophoblast cell-surface marker; CEA, carcinoembryonic antigen; CA, carbohydrate antigen

Key words: colon cancer, trophoblast cell-surface marker 2, therapeutic target, metastasis, Dukes stage

\section{Introduction}

Colorectal cancer is one of the leading causes cancer-associated mortality and morbidity in several countries (1). Colorectal cancer, a highly prevalent cancer in males and females, includes colon cancer and rectal cancer, due to their adjacent anatomical locations. Colon cancer is the second most commonly diagnosed type of cancer in females and the third in males worldwide (2). Carethers et al demonstrated a higher prevalence for microsatellite instability (MSI) among cancers patients of African American ethnicity, and the MSI prevalence in these cancer patients was observed to be half that of Caucasian patients without changes in $\mathrm{CD}^{+} \mathrm{T}$ cell infiltration, which may contribute towards the higher mortality rates in the former group from colon cancer (3). Several tumor biomarkers, including carcinoembryonic antigen (CEA), carbohydrate antigen (CA) 19.9 and CA 12.5, have been detected in a number of patients with colon cancer (4). The levels of CEA and CA19.9 are often increased in advanced colon cancer (5) and have been considered as an early biological signal of colon cancer recurrence (6). Increasing current knowledge of cancer cell proliferation, migration and invasion are central to understanding tumor progression and metastasis. The local tumor environment may provide specific environmental cues to alter the behavior of the cells and promote metastasis.

For the last two decades, the liver has been the most frequent site of colon cancer metastasis (7), which affects the efficacy of treatment and the prognosis of patients. Therefore, it is important that the pathogenesis and treatment of colon cancer is addressed.

Human trophoblast cell-surface marker (TROP-2) is a cell surface glycoprotein, which was originally identified in human placental trophoblasts and has been reported to be highly expressed in various types of human carcinoma, including oral (8), lung (9), pancreatic (10) and gastric carcinoma (11). By contrast, the expression of TROP-2 in adult somatic tissues is minimal or absent (12). TROP-2, as a type I transmembrane protein, has been cloned from human (13) and mouse cells (14), and the overexpression of TROP-2 cell surface protein was correlated with aggressive behavior and poor prognosis in certain types of epithelial carcinoma by Coldren et al (15). It has been reported that TROP-2 is important in determining the fate of tumor growth, and the expression of TROP-2 may 
be necessary for anchorage-independent growth, invasiveness and tumorigenesis in cancer cells (16). Notably, previous studies have suggested that overexpression of TROP-2 is associated with increased tumor aggressiveness and metastasis, and decreased patient survival rate (17). Therefore, there is a increasing interest in TROP-2 as a potential therapeutic target for solid types of cancer (18). For example, hRS7, a humanized anti-TROP-2 antibody, is currently under clinical trial as a drug for patients with advanced epithelial cancer (19).

The functional role of TROP-2 in cancer remains to be fully elucidated. Several studies have demonstrated that the TROP-2 gene encodes a tumor-associated calcium signal transducer, which is involved in the regulation of cell-cell adhesion, and an antibody against TROP-2 has been observed to alter intracellular calcium levels $(20,21)$. Other studies have suggested that TROP-2 is highly expressed in certain developing tissues, it may be important in morphogenesis $(18,22,23)$. Notably, evidence has revealed that humans born with homozygous inactivating mutations in TROP-2 exhibited only limited pathology (24). The biological function of TROP-2 is focused on promoting self-renewal and hyperplasia in the prostate, which has been attributed to the accumulation of the intracellular domain of TROP-2 in the nucleus, following its cleavage through regulating intramembrane proteolysis (25). Additionally, reports suggest that prostate basal cells overexpressing TROP-2 possess stem cell capacities, including tissue regeneration, self-renewal and multilineage differentiation $(25,26)$.

To the best of our knowledge, the expression and biological function of TROP-2 in the tumorigenesis and invasiveness of colon cancer has not been investigated. Therefore, the purpose of the present study was to investigate the expression of TROP-2 in colon cancer tissues, and to assess its biological and clinical significance.

\section{Patients and methods}

Patients. Cancer tissues and tumor-adjacent tissues, the latter sited $5 \mathrm{~cm}$ from the cancer tissue edge and confirmed as normal tissue by a pathologist, were obtained from 82 patients diagnosed with colon cancer, who had not received radiation, chemotherapy or immunotherapy prior to surgery. Normal tissue specimens were collected from 30 patients with non-neoplastic colon mucosa. All specimens were obtained from the Department of General Surgery, Hebei United University Affiliated Hospital (Hebei, China), between January 2012 and December 2013. Eligible cases were between the ages of 35 and 90 years $(59.8 \pm 5.7)$, included 53 male cases and 30 female cases, and had pathologically confirmed invasive adenocarcinoma of the colon. Additional clinicopathologic features of the colon cancer are listed in Table 1. Under sterile conditions, tissues samples of $0.5 \mathrm{~cm}$ diameter were obtained and shock-frozen in liquid nitrogen. The present study was approved by the Ethics Committee of Hebei Medical University (Shijiazhuang, China), and written informed consent was obtained from all patients enrolled.

Western blot analysis. Total proteins were extracted from the tissues with radioimmunoprecipitation assay buffer (Cell Signaling Technology, Inc., Danvers, MA, USA) with the addition of Halt Protease Inhibitor Cocktail (Cell
Signaling Technology, Inc.), and protein concentration was determined using a bicinchoninic acid reagent (Solarbio, Beijing, China) method. The samples were then subjected to $10 \%$ sodium dodecyl sulfate polyacrylamide gel electrophoresis (SDS-PAGE). The separated proteins on the gel were transferred onto polyvinylidene difluoride membranes (Roche Diagnostics, Mannheim, Germany). The blots were blocked with $5 \%$ fat-free dry milk for $1 \mathrm{~h}$ at room temperature. Subsequently, the blots were incubated overnight at $4^{\circ} \mathrm{C}$ with the following primary antibodies: Rabbit anti-human TROP-2 polyclonal antibody (cat. no., sc-80406; dilution, 1:400; Santa Cruz Biotechnology, Inc., Santa Cruz, CA, USA) and rabbit anti-human $\beta$-actin monoclonal antibody (cat. no., sc-130657; dilution, 1:600; Santa Cruz Biotechnology, Inc.). The blots were then incubated with horseradish peroxidase-conjugated anti-rabbit immunoglobulin ( $\mathrm{IgG} ; 1: 5,000$; Cell Signaling Technology, Inc.) for $1 \mathrm{~h}$ at room temperature. The immunoblot on the membrane was visible following development using an enhanced chemiluminescence detection system (ChemiDoc XRS; Bio-Rad Laboratories, Inc., Hercules, CA, USA), and the densitometric signals were quantified using an imaging program. The immunoreactive bands of all the proteins expressed were normalized to the intensity of corresponding bands for $\beta$-actin. The densities of the western blotting bands were quantified using National Institutes of Health ImageJ software, version 1.41 (Bethesda, MD, USA).

Immunofluorescence analyses. All specimens were fixed in $4 \%$ paraformaldehyde for $24 \mathrm{~h}$, following $30 \%$ sucrose solution in $0.1 \mathrm{M}$ PBS ( $\mathrm{pH} 7.4$ was added until the cells were observed to sink to the bottom, when they were then embedded in OCT. Frozen sections $(10 \mu \mathrm{m})$ were sliced using a frozen slicer, treated with $0.4 \%$ Triton- 100 for $15 \mathrm{~min}$, and blocked in normal donkey serum for $1 \mathrm{~h}$. The frozen sections were then incubated with rabbit anti-human TROP-2 polyclonal antibody (1:50; Santa Cruz Biotechnology, Inc.), overnight at $4^{\circ} \mathrm{C}$. The next day, the sections were incubated with a mixture of fluorescein-conjugated horse anti-rabbit IgG (cat. no., SA00003-8; dilution, 1:1,000; Cell Signaling Technology, Inc) for $2 \mathrm{~h}$ at $37^{\circ} \mathrm{C}$ in the dark. The nuclei were stained using 4,6-diamino-2-phenylindole (DAPI; Wuhan Boster Biological Engineering, Wuhan, China). Images were captured under a laser scanning confocal microscope (Olympus FV1000: Olympus Corporation, Tokyo, Japan). Primary antibodies were replaced with PBS in the negative control group.

Statistical analysis. All experiments were repeated three times and similar results were obtained. Statistical analysis was performed using SPSS 16.0 statistical software (SPSS, Inc., Chicago, IL, USA). Data are expressed as the mean \pm standard error of the mean. Statistical analysis was performed using one-way analysis of variance, Student's t-test and a $\chi^{2}$ test. $\mathrm{P}<0.05$ was considered to indicate a statistically significant difference.

\section{Results}

Expression of TROP-2 in colon cancer, tumor-adjacent and normal tissues. The expression levels of TROP-2 in 
Table I. Association between the expression of TROP-2 in and clinicopathological features in patients with colon cancer.

\begin{tabular}{|c|c|c|c|c|}
\hline Factor & $\mathrm{n}$ & TROP-2(+) & TROP-2(-) & P-value \\
\hline \multicolumn{5}{|l|}{ Gender } \\
\hline Male & 52 & 48 & 4 & \multirow[t]{2}{*}{0.512} \\
\hline Female & 30 & 27 & 3 & \\
\hline \multicolumn{5}{|c|}{ Age (years) } \\
\hline$\leq 59$ & 39 & 34 & 5 & \multirow[t]{2}{*}{0.684} \\
\hline$>59$ & 43 & 41 & 2 & \\
\hline \multicolumn{5}{|c|}{ Differentiation } \\
\hline High & 24 & 21 & 3 & \multirow[t]{3}{*}{0.857} \\
\hline Middle & 33 & 30 & 3 & \\
\hline Low & 25 & 24 & 1 & \\
\hline \multicolumn{5}{|c|}{ Lymph node metastases } \\
\hline Yes & 54 & 51 & 3 & \multirow[t]{2}{*}{0.018} \\
\hline No & 28 & 24 & 4 & \\
\hline \multicolumn{5}{|c|}{ Dukes stage } \\
\hline $\mathrm{A}+\mathrm{B}$ & 38 & 33 & 5 & \multirow[t]{2}{*}{0.002} \\
\hline $\mathrm{C}+\mathrm{D}$ & 44 & 42 & 2 & \\
\hline
\end{tabular}

TROP-2, human trophoblast cell-surface marker; +, positive expression; -, negative expression.

colon cancer tissues, tumor-adjacent tissues and normal tissues were examined using western blot analysis (Fig. 1). Notably, in contrast to the colon cancer tissues, the normal tissues exhibited decreased protein expression of Trop-2. The immunoreactivity for TROP-2 in the tumor-adjacent tissues was higher than in the normal tissues, however, this difference was not statistically significant $(\mathrm{P}>0.05)$. However, the expression of TROP-2 in colon cancer tissues was significantly higher than that in the tumor-adjacent tissues and normal tissues $(\mathrm{P}<0.05)$. These result demonstrated that TROP-2 was highly expressed in colon cancer and may offer potential as an important biomarker for early diagnosis in colon cancer.

Expression of TROP-2 in colon cancer tissues with/ without lymph node metastases. As shown in Fig. 2, positive expression of TROP-2 was observed in the colon cancer with lymph node metastasis group, which was significantly higher, compared with that in the non-lymph node metastasis group $(\mathrm{P}<0.05)$. This indicated that the protein expression of TROP-2 was associated with lymph node metastasis in colon cancer.

Expression of TROP-2 in colon cancer tissues of different Dukes stages. The expression of Trop-2 in the tissue samples of the Dukes A, Dukes B, Dukes C and Dukes D stages were analyzed using western blot analysis. As shown in Fig. 3, the expression of, TROP-2 expression was upregulated in the Dukes A stage cancer tissues, and the level of expression increased with the increasing Dukes stage. These differences were statistically significant $(\mathrm{P}<0.05)$ and these findings indicated that TROP-2 was associated with the invasion and metastasis of colon cancer.

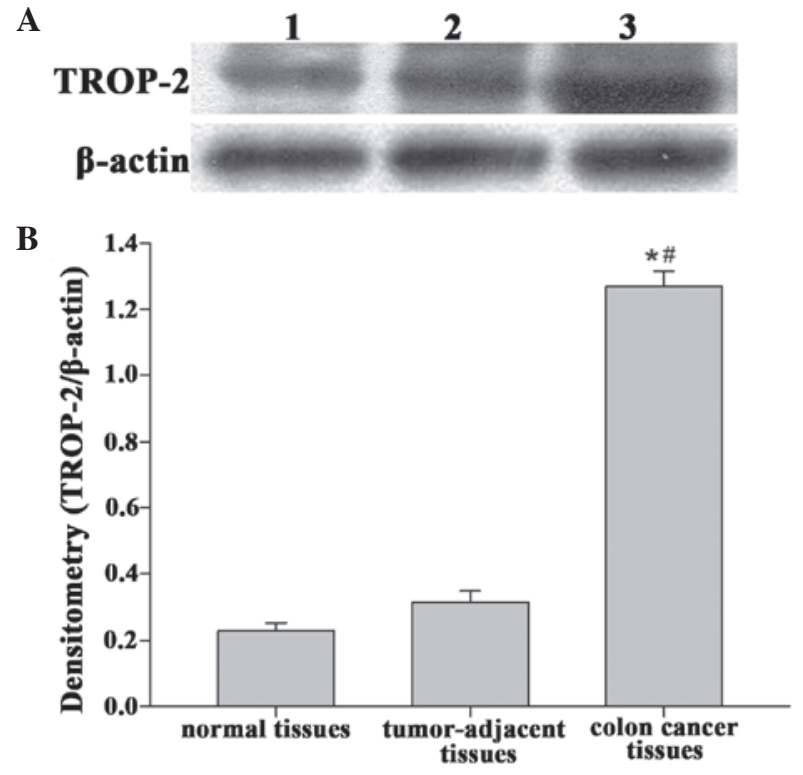

Figure 1. TROP-2 protein is expressed in colon cancer tissues. (A) Western blot analysis of the protein expression of TROP-2. 1, normal tissues; 2 , tumor-adjacent tissues; 3 , colon cancer tissues. (B) Densitometric analysis of the protein expression of TROP- 2 relative to $\beta$-actin. The optical densities of the respective protein bands were analyzed using ImageJ software. The quantitative results of the protein expression levels of TROP-2 are expressed as the mean \pm standard error of the mean. The results demonstrated that the expression of TROP-2 was markedly increased in colon cancer tissues, compared with the normal tissues and the tumor-adjacent tissues. ${ }^{*} \mathrm{P}<0.05$, vs. normal tissues; ${ }^{\#} \mathrm{P}<0.05$, vs. tumor-adjacent tissues. TROP-2, human trophoblast cell-surface marker.

Confirmation of the protein expression of TROP-2 using immunofluorescence analyses. Further experiments were performed to evaluate the localization of the TROP-2 protein 


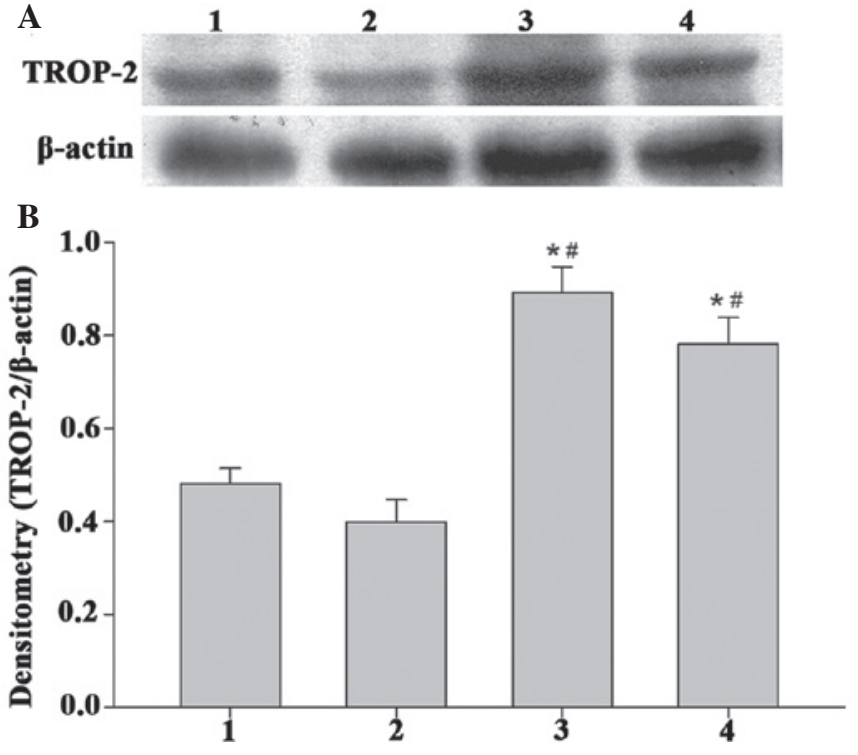

Figure 2. Correlation between the expression of TROP-2 and lymph node metastases. The protein levels of TROP-2 in the cancer tissues were detected using (A) immunoblotting and (B) densitometric analysis of the TROP-2 band relative to $\beta$-actin. 1 and 2 , colon cancer tissues without lymph node metastases; 3 and 4, colon cancer tissues with lymph node metastases. The results demonstrated that the protein expression of TROP-2 was markedly increased in the colon cancer tissues with lymph node metastases, compared with the colon cancer tissues without lymph node metastasis. Data are expressed as the mean \pm standard error of the mean. ${ }^{*} \mathrm{P}<0.05$ in group 1 , and ${ }^{~} \mathrm{P}<0.05$ in group 2, vs. colon cancer tissues without lymph node metastases. TROP-2, human trophoblast cell-surface marker.
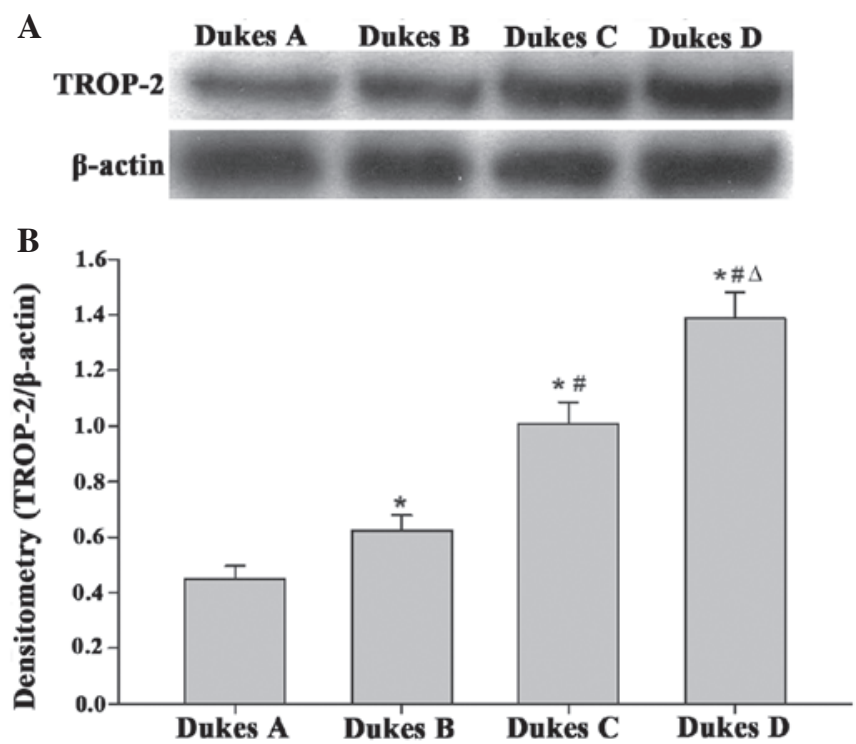

Figure 3. Expression of TROP-2 in colon cancer tissues of different Dukes stages. (A) Western blot analysis of the protein expression of TROP-2 in cancer tissues of different Dukes stages. (B) Densitometric analyses of the protein expression of TROP-2 relative to $\beta$-actin. The results demonstrated that the protein expression of TROP-2 was significantly increased with increasing Dukes stage. Data are expressed as the mean \pm standard error of the meam. ${ }^{*} \mathrm{P}<0.05$, vs. Dukes A; ${ }^{\#} \mathrm{P}<0.05$, vs. Dukes $\mathrm{B} ;{ }^{\Delta} \mathrm{P}<0.05$, vs. Dukes $\mathrm{C}$ TROP-2, human trophoblast cell-surface marker.

in colon cancer tissues. TROP-2 protein in cancer tissues of different Dukes stages was detected using immunofluorescence staining. As shown in Fig. 4, TROP-2 protein was stained with rabbit anti-TROP-2 antibody and green fluorescent protein-labeled secondary antibody. TROP-2 protein was located in the cell membrane surface of the colon cancer cells. The TROP-2-immunoreactive structures appeared green and circular, and the nuclei were stained with DAPI, observed as red fluorescence. The cells, which appeared green in the centre and surrounded by red subsequent to merging, were observed under a laser scanning confocal microscope. The results also demonstrated that the expression of TROP-2 was upregulated with increasing Dukes stage.

Correlations between the expression of TROP-2 and clinical pathological features of colon cancer. The association between the expression of TROP-2 and the clinicopathological features of colon cancer patients were analyzed using a $\chi^{2}$ test. As shown in Table I, the high protein expression level of TROP-2 in colon cancer was associated with lymph node metastasis and Dukes classification $(\mathrm{P}=0.018$ and $\mathrm{P}=0.002$, respectively). By contrast, no statistically significant correlation was observed with gender, age or the degree of differentiation. These results indicated that the protein expression of TROP-2 protein is important in the development and metastasis of colon cancer.

\section{Discussion}

Colon cancer remains a major public health concern worldwide, and presents a considerable disease burden. Despite increasing knowledge of the cellular and molecular signaling pathways underlying colon cancer, therapeutic outcomes remain only moderately successful, with poor efficacy and marked variation in therapeutic outcomes among patients. At present, surgical resection remains the only curative treatment option, however, this involves costly and invasive procedures with certain considerable limitations. Despite efforts to determine its molecular mechanism, the precise mechanisms underlying the malignant outcomes of colon cancer remain to be elucidated. There are certain risk factors associated with colon cancer, including inflammatory syndromes, hereditary factors and environmental factors (27). Biologically active molecules, which are involved in aggressiveness and metastasis are important for the mechanism of tumor dissemination (28). Therefore, examining gene and protein changes associated with the invasion and metastasis of colon cancer is important for identifying the molecular targets for clinical treatment.

In the present study, the protein expression of TROP-2 was analyzed in patients with colon cancer. A significant upregulation in the protein expression of TROP-2 was observed in colon cancer, relative to the corresponding normal tissues and tumor-adjacent tissues. However, whether the overexpression of TROP-2 is actively involved in tumorigenesis, and whether targeting TROP-2 is of therapeutic use remains to be elucidated. The present study investigated the expression of TROP-2 in colon cancer with/without lymph node metastases and in tissues exhibiting different Dukes stages. In addition, the correlation between the expression of TROP-2 and clinical factors were examined to determine its clinicopathological significance in colon cancer. The findings revealed that overexpression of TROP-2 protein in colon cancer was significantly 


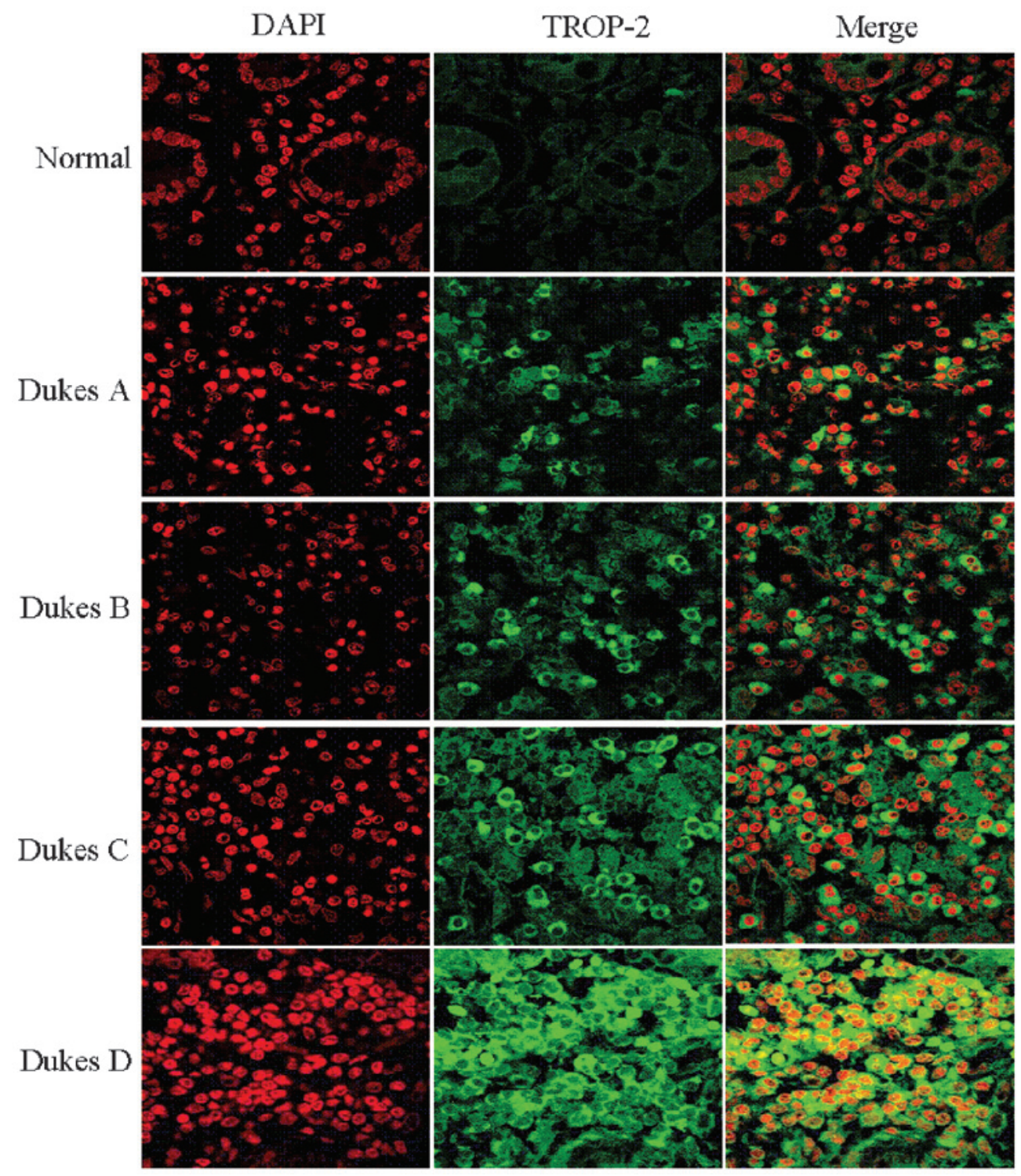

Figure 4. Localization of TROP-2 protein in colon cancer tissues of different Dukes stages. The nuclei (red) and TROP-2 immunoreactivity (green) were observed in the confocal images. The results revealed that TROP-2 protein was localized predominantly in the cell membrane and that expression of TROP-2 increased in line with Dukes stages. All microphotographs were visualized using confocal laser scanning microscopy (magnification, x1,200). TROP-2, human trophoblast cell-surface marker.

associated with lymph node metastasis and Dukes classification, suggesting the overexpression of TROP-2 in colon cancer tissue could induces mucosal instability, premalignant polyps and malignant transformation. TROP-2 was observed to be necessary for the tumorigenesis and invasiveness of colon cancer, and these effects may be reduced effectively with an antibody against the extracellular domain of TROP-2. Notably, these findings led to the hypothesis that antibodies against TROP-2 protein may inhibit the tumorigenesis of colon cancer, presenting a novel anticancer target. TROP-2 has also been suggested as a target for anticancer immunotherapy in other types of epithelial carcinoma (28). Anti-TROP-2 monoclonal or polyclonal antibodies have been observed to destroy uterine serous papillar carcinoma cells through Antibody-dependent cell-mediated cytotoxicity in vitro (19). Therefore, in contrast to previous studies, which focused predominantly on the oncogenic effects of Trop-2, the results of the present study demonstrated that TROP-2 is functionally important in tumorigenesis, and present established biological markers of colon cancer. Given its accessibility to antibodies as a cell surface protein and its selective expression in tumor cells, TROP-2 may be a potential therapeutic target for colon cancer. Additionally, the restricted expression of TROP-2 in normal tissues suggested that anti-TROP-2 therapeutics may be of limited cytotoxicity.

In conclusion, the results of the present study indicated for the first time, to the best of our knowledge, that TROP-2 protein is overexpressed in colon cancer tissues. Furthermore, correlation exists between the expression of Trop- 2 and the pathogenesis of colon cancer. The TROP-2 cell surface protein may regulate the growth, invasion and metastasis of tumor cells. These findings provide novel insight into the role of TROP-2 in human colon cancer, as a novel, widespread stimulator of colon cancer growth and a unique biomarker. Therefore, targeting the overexpression of TROP-2 using immunotherapeutic strategies may offer an attractive and 
potentially effective approach in the treatment of patients with colon cancer.

\section{Acknowledgements}

The present study was supported by a grant from the Science and Technology Development Project of Tangshan City (grant no. 10140201A-3). The authors would like to thank Dr Jian-Hui Cai for their critical evaluation of the manuscript, the members of the Hebei United University Laboratory for providing technical assistance, and our colleagues for their assistance during the course of the study.

\section{References}

1. Chiang EP, Tsai SY, Kuo YH, et al: Caffeic Acid Derivatives Inhibit the Growth of Colon Cancer: Involvement of the PI3-K/Akt and AMPK Signaling Pathways. PloS One 9: e99631, 2014.

2. Boncheva V, Bonney SA, Brooks SE, et al: New targets for the immunotherapy of colon cancer-does reactive disease hold the answer? Cancer Gene Ther 20: 157-168, 2013.

3. Carethers JM, Murali B, Yang B, et al: Influence of Race on Microsatellite Instability and CD8+ T Cell Infiltration in Colon Cancer. PloS One 9: e100461, 2014.

4. Selcukbiricik F, Bilici A, Tural D, Erdamar S, Soyluk O, Buyukunal E, Demirelli F and Serdengecti S: Are high initial CEA and CA 19-9 levels associated with the presence of K-ras mutation in patients with metastatic colorectal cancer? Tumour Biol 34: 2233-2239, 2013.

5. Mayer RJ, Garnick MB, Steele GD Jr. and Zamcheck N: Carcinoembryonic antigen (CEA) as a monitor of chemotherapy in disseminated colorectal cancer. Cancer 42: 1428-1433, 1978.

6. Petrioli R, Licchetta A, Roviello G, et al: CEA and CA19.9 as early predictors of progression in advanced/metastatic colorectal cancer patients receiving oxaliplatin-based chemotherapy and bevacizumab. Cancer Invest 30: 65-71, 2012.

7. Van den Eynden GG, Majeed AW, Illemann M, et al: The multifaceted role of the microenvironment in liver metastasis: biology and clinical implications. Cancer Res 73: 2031-2043, 2013.

8. Fong D, Spizzo G, Gostner JM, et al: TROP2: a novel prognostic marker in squamous cell carcinoma of the oral cavity. Modern Pathol 21: 186-191, 2008.

9. Pak MG, Shin DH, Lee CH and Lee MK: Significance of EpCAM and TROP2 expression in non-small cell lung cancer. World J Surg Oncol 10: 53, 2012.

10. Fong D, Moser P, Krammel C, et al: High expression of TROP2 correlates with poor prognosis in pancreatic cancer. $\mathrm{Br}$ J Cancer 99: 1290-1295, 2008.

11. Mühlmann G, Spizzo G, Gostner J, et al: TROP2 expression as prognostic marker for gastric carcinoma. J Clin Pathol 62: 152-158, 2009.
12. Zhang L, Zhou W, Velculescu VE, et al: Gene expression profiles in normal and cancer cells. Science 276: 1268-1272, 1997.

13. Fornaro M, Dell'Arciprete R, Stella M, et al: Cloning of the gene encoding Trop-2, a cell-surface glycoprotein expressed by human carcinomas. Int J Cancer 62: 610-618, 1995.

14. El Sewedy T, Fornaro M and Alberti S: Cloning of the murine TROP2 gene: conservation of a PIP2-binding sequence in the cytoplasmic domain of TROP-2. Int J Cancer 75: 324-330, 1998.

15. Coldren CD, Helfrich BA, Witta SE, et al: Baseline gene expression predicts sensitivity to gefitinib in non-small cell lung cancer cell lines. Mol Cancer Res 4: 521-528, 2006.

16. Bignotti E, Todeschini P, Calza S, et al: Trop-2 overexpression as an independent marker for poor overall survival in ovarian carcinoma patients. Eur J Cancer 46: 944-953, 2010.

17. Ambrogi F, Fornili M, Boracchi $\mathrm{P}$, et al: Trop-2 is a determinant of breast cancer survival. PloS One 9: e96993, 2014.

18. Cubas R, Li M, Chen C and Yao Q: Trop2: a possible therapeutic target for late stage epithelial carcinomas. Biochim Biophys Acta 1796: 309-314, 2009.

19. Varughese J, Cocco E, Bellone S, et al: Uterine serous papillary carcinomas overexpress human trophoblast-cell-surface marker (Trop-2) and are highly sensitive to immunotherapy with hRS7, a humanized anti-Trop-2 monoclonal antibody. Cancer 117: 3163-3172, 2011.

20. Ripani E, Sacchetti A, Corda D and Alberti S: Human Trop-2 is a tumor-associated calcium signal transducer. IntJ Cancer 76: 671-676, 1998.

21. Trerotola M, Li J, Alberti S and Languino LR: Trop-2 inhibits prostate cancer cell adhesion to fibronectin through the betal integrin-RACK1 axis. J Cell Physiol 227: 3670-3677, 2012.

22. Trerotola M, Cantanelli P, Guerra E, et al: Upregulation of Trop-2 quantitatively stimulates human cancer growth. Oncogene 32: 222-233, 2013.

23. Huang HY: Role of TROP2 in cancer and as potential therapeutic target. Zhonghua Bing Li Xue Za Zhi 42: 860-863, 2013 (Article in Chinese).

24. Lu J, Izvolsky KI, Qian J and Cardoso WV: Identification of FGF10 targets in the embryonic lung epithelium during bud morphogenesis. J Biol Chem 280: 4834-4841, 2005.

25. Stoyanova T, Goldstein AS, Cai H, Drake JM, Huang J and Witte ON: Regulated proteolysis of Trop2 drives epithelial hyperplasia and stem cell self-renewal via beta-catenin signaling. Genes Dev 26: 2271-2285, 2012.

26. Goldstein AS, Lawson DA, Cheng D, Sun W, Garraway IP and Witte ON: Trop2 identifies a subpopulation of murine and human prostate basal cells with stem cell characteristics. Proc the Natl Acad Sci USA 105: 20882-20887, 2008.

27. Antonic V, Stojadinovic A, Kester KE, Weina PJ, Brücher BL, Protic M, Avital I, and Izadjoo M: Significance of infectious agents in colorectal cancer development. J Cancer 4: 227-240, 2013.

28. Messick CA, Church J, Casey G and Kalady MF: Identification of the methylator (serrated) colorectal cancer phenotype through precursor serrated polyps. Dis Colon Rectum 52: 1535-1541, 2009. 